\title{
Assessing the Role of Network Topology in Transportation Network Resilience X. Zhang ${ }^{1}$ E. Miller-Hooks ${ }^{2}$ $\&$ K. Denny ${ }^{3}$
}

Originally submitted November 30, 2013

Revision 1: March 3, 2015

Revision 2: May 4, 2015

The abstract representation of a transportation system as a network of nodes and interconnecting links, whether that system involves roadways, railways, sea links, airspace, or intermodal combinations, defines a network topology. Among the most common in the context of transportation systems are the grid, ring, hub-and-spoke, complete, scale-free and small-world networks. This paper investigates the role of network topology, and the topology's characteristics, in a transportation system's ability to cope with disaster. Specifically, the paper hypothesizes that the topological attributes of a transportation system significantly affect its resilience to disaster events. Resilience accounts for not only the innate ability of the system to absorb externally induced changes, but also cost-effective and efficient, adaptive actions that can be taken to preserve or restore performance post-event. Comprehensive and systematically designed numerical experiments were conducted on 17 network structures with some relation to transportation system layout. Resilience of these network structures in terms of throughput, connectivity or compactness was quantified. Resilience is considered with and without the benefits of preparedness and recovery actions. The impact of component-level damage on system resilience is also investigated. A comprehensive, systematic analysis of results from these experiments provides a basis for the characterization of highly resilient network topologies and conversely identification of network attributes that might lead to poorly performing systems.

Key words: Resilience, vulnerability, reliability, network performance, infrastructure systems

1 Graduate Research Assistant, Department of Civil and Environmental Engineering, 1173 Glenn Martin Hall, University of Maryland, College Park, Maryland 20742, (301)-405-7768, xzhang@umd.edu

2 Corresponding author, Professor, Department of Civil and Environmental Engineering, 1173 Glenn Martin Hall, University of Maryland, College Park, Maryland 20742, (301)-405-2046, elisemh@umd.edu

3 Undergraduate Research Assistant, Department of Civil and Environmental Engineering, 1173 Glenn Martin Hall, University of Maryland, College Park, Maryland 20742, kdenny1@ umd.edu 


\section{Introduction}

The abstract representation of a transportation system as a network of nodes and interconnecting links, whether that system involves roadways, railways, sea links, airspace, or intermodal combinations, defines a network topology. Such topologies may have regular or irregular shape, and many topologies have been generically categorized. Among the most common in the context of transportation systems are the grid, ring, hub-and-spoke, complete, scale-free and small-world networks. Many arterial roadway networks have a grid or ring shape, networks of towns can be well-represented by small-world networks, while air systems are commonly shaped as hub-andspoke networks. These networks can be characterized by various measures, and even networks with different topologies can have common characteristics. This paper investigates the role of network topology, and the topology's characteristics, in a transportation system's ability to cope with disaster. Specifically, the paper hypothesizes that the topological attributes of a transportation system significantly affect its resilience to disaster events. The impact of component (or local) damage on system performance is also investigated.

In this study, a definition of resilience given in (Miller-Hooks et al., 2012) is adapted that explicitly considers the system's coping capacity, along with the effects of pre-disaster preparedness and adaptive response actions that can be quickly taken in the disaster's aftermath while adhering to a fixed, small budget and short duration of time for implementing recovery options. The system's coping capacity is measured through its capability to resist and absorb disaster impact through redundancies, excess capacities. The concept of resilience differs from that of similar, more commonly employed performance measures, such as vulnerability, in that resilience accounts for not only the ability of the network to cope with a disruptive event, but the impact of adaptive actions that can be taken to ameliorate damage impact.

Insights gleaned from results of systematically designed numerical experiments on 17 generic network structures provide a basis for the characterization of highly resilient network topologies and conversely identification of network attributes that might lead to poorly performing systems. In the assessment, three resilience measures based on throughput, connectivity and compactness (by way of average reciprocal distance) are considered with and without the benefits of preparedness $(p)$ and recovery actions $(r)$. 
Preliminary experiments involving four carefully designed 10-node complete, hub-based, grid and random networks were completed (Chen and Miller-Hooks, 2012). A concept of resilience in which recovery actions were possible was tested. However, no preparedness options that can improve a network's coping capacity and support recovery actions were considered in the study. Results of these runs indicated that topological structures with limited redundancies faired worst when no recovery actions were supported; however, even with limited or modest budgets to support recovery options, improvements in resilience levels were achieved. It was also noted that improvements were greatest for networks with hubs. This is because exercising only a few options could restore connectivity between a large number of O-D pairs. Network structures that traditionally fare poorly when considering only the network's coping capacity (i.e. where no budget is available for response actions), performed well by focusing recovery actions on the most critical links. These experiments involved very small networks of only four topological classifications applying only one concept of resilience. A more comprehensive analysis from which significantly deeper and broader insights can be garnered is presented herein.

The studied network topologies are introduced in the next section. Measures for their characterization, such as diameter, betweeness centrality and the Shimbel index, are also discussed. This is followed by methods for measuring maximum resilience with respect to the chosen throughput, connectivity and compactness metrics. The experimental design, numerical results and analysis follow. Finally, conclusions and implications of the findings for transportation applications are discussed.

\section{Literature Review}

Many works have proposed measures to characterize networks and their performance for a range of applications, including physics, geography, the Internet, and biological and social systems. Early examples include (Kansky, 1963, Haggett and Chorley, 1967 and Garrison and Marble, 1974). Kansky (1963) considered nodal importance and network complexity in transportation networks with three main indices: Alpha, Beta, and Gamma indices, all measures of connectivity. These and other measures are defined in Table 1 of the next section. Their studies, however, were hampered by limited computational resources. 
More recently, a number of works have investigated relationships between network shape and transportation system layout, including, for example, road and air networks (e.g., Gastner and Newman, 2006; Reggiani et al., 2011) and subway networks (e.g., Derrible and Kennedy, 2010). Random, scale-free and small-world network structures were found to be particularly relevant as demonstrated through the following example works of this type. In random graphs, nodes are randomly linked with an equal probability of placing a link between any pair of nodes. As defined in (Barabási and Albert, 1999), a scale-free network has a nodal degree distribution that follows a power law. Thus, some nodes have a degree that greatly exceeds the average. Small-world networks, on the other hand, are densely connected in local regions, creating highly connected subgraphs with few crucial connections between distant neighbors. Wu et al. (2004) showed that scale-free type characteristics exist in urban transit networks in Beijing, while Latora and Marchiori (2002) suggested that the Boston subway system has a small-world network structure. Watts and Strogatz (1998) studied the performance of neural and power grid networks in terms of shortest average path length and clustering. They found that some neural and power grid networks have the shape of small-world networks. Zhao and Gao (2007) studied the performance of small-world, scale-free and random networks in terms of total travel time and traffic volume in the context of a traffic network.

Other works have studied connections between system topology and performance. In the context of transit networks, Li and Kim (2014), for example, proposed a connectivity-based survivability measure to study the Beijing subway system. Similarly, Rodríguez-Núñez and García-Palomares (2014) presented a vulnerability measure and applied it to study the Madrid Metro. In work by Derrible and Kennedy (2010), the robustness of 33 metro systems around the world was investigated. In their work, robustness is defined in terms of cyclicity. Cyclicity is a connectivity measure that like average degree is used to characterize a network topology herein. Exploiting noted relationships between these real system layouts and scale-free and small-world network structures, they provided strategies for improving performance of both small and large systems. They provide a comprehensive review of related works, as well. O'Kelly (forthcoming) discusses the role of hubs in network vulnerability and resilience of various network structures. Finally, Reggiani et al. (2015) propose the use of connectivity as a unifying framework for considering resilience and vulnerability in relation to transport networks. They test this concept through a synthesis of related literature. Numerous additional articles consider the performance 
of specific transportation networks under various resilience-related measures, but they do not investigate the general role of network topology.

Different from earlier works that studied relationships between network topology and vulnerability or similar measures, this paper investigates the role of network topology in system resilience using a definition of resilience that accounts not only for the network's inherent coping capacity, but also its ability to efficiently adapt post-event.

\section{Network Resilience Measurement}

\subsection{Selection and Characterization of Network Topologies}

The 17 network topologies that were investigated were chosen from approximately 25 topologies discovered in a search of the literature covering many areas, including transportation, communications, the Internet, general graph theory and biological systems, among others, but with a specific emphasis on topologies of relevance to transportation systems. Many were abstracted from existing transportation system layouts.

The basic structure of each of the chosen network classes is described in Figure 1. These basic structures provide the fundamental elements for the construction of larger comparable networks. This extrapolation to larger network sizes (greater number of nodes and links) is also included in Figure 1. The resilience of each basic structure and structures constructed from these elements (used as tiles where logical to do so) is studied. 

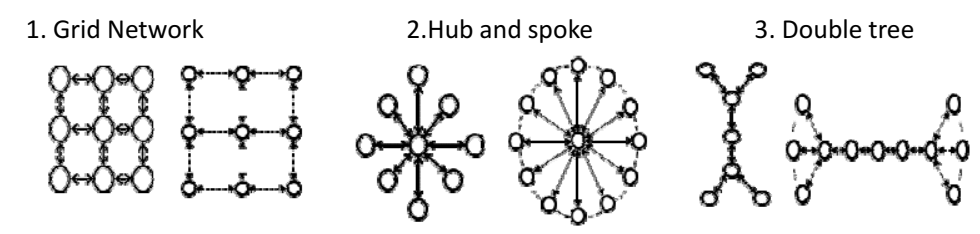

4. Ring network

5. Matching pairs

6. Complete
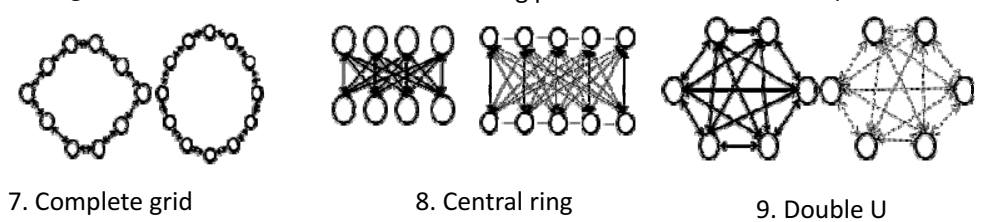

8. Central ring
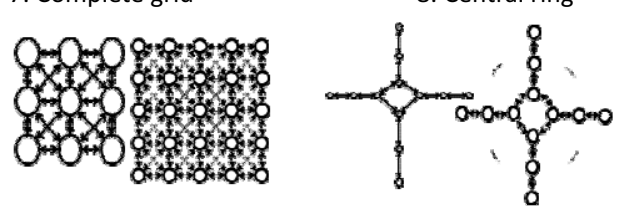

9. Double U
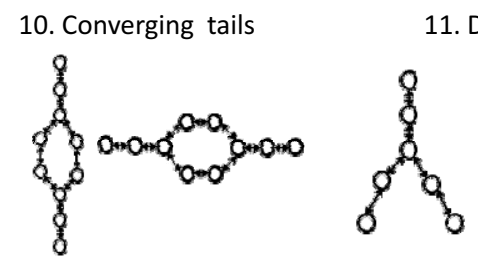

11. Diverging tails
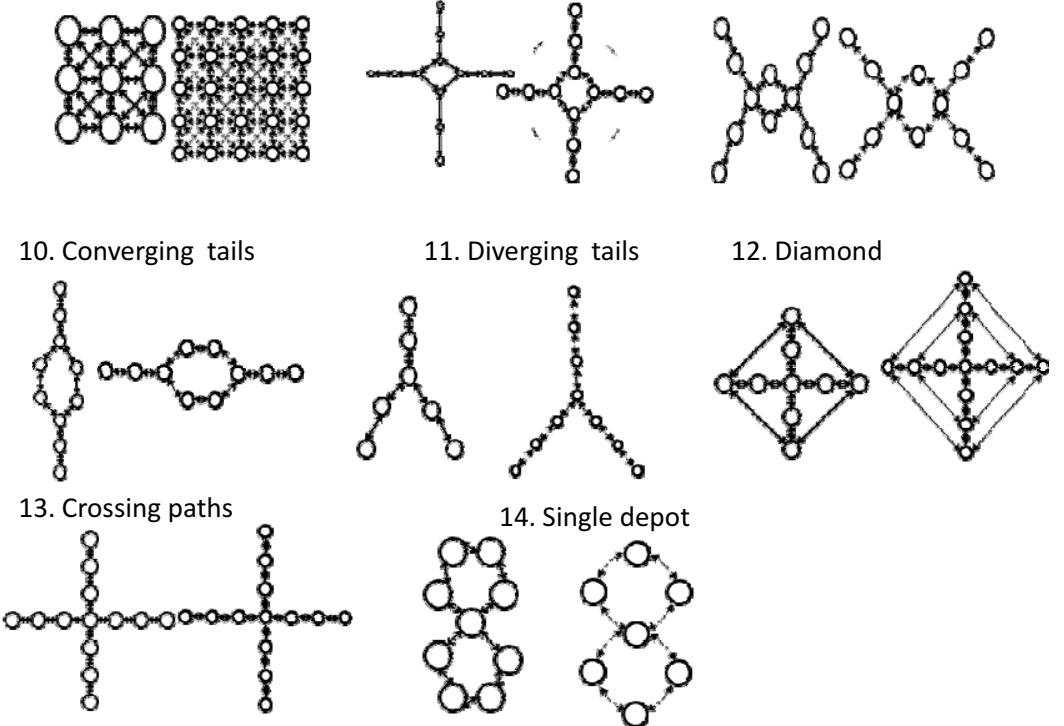

14. Single depot
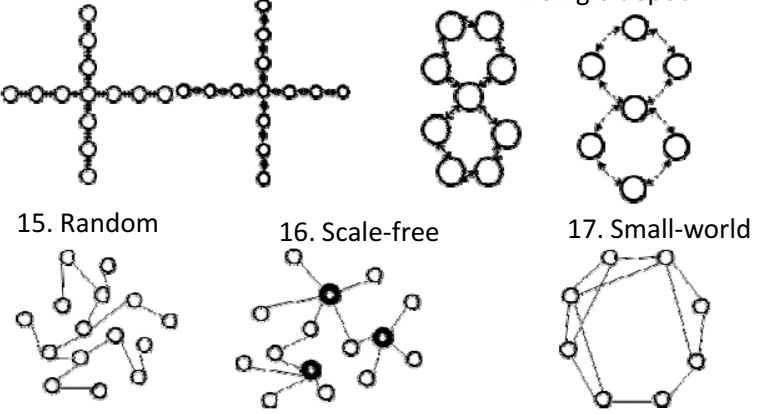

Figure 1 Network topology and extrapolation

A network topology can be characterized in terms of commonly used connectivity and accessibility measures such as those listed in Table 1 (see Grubesic et al. (2008) for a detailed overview of these graph theoretic measures and their application to a case study involving vulnerability assessment and critical arc/node identification for the Internet). Connectivity measures are used to assess redundancies and connectedness, while accessibility measures are used to compare the relative position of nodes in the network. Such measures have been widely applied. For example, Perna et al. (2008) measured communication efficiency within a network of termite nests using measures such as average path length, clustering coefficient, betweenness 
centrality, and local graph redundancy. Such characterization of the topologies enables consistency in comparisons and generalization of the findings.

Table 1 Typical graph-theoretic network measures

\begin{tabular}{|c|c|c|c|}
\hline \multicolumn{4}{|l|}{ Connectivity } \\
\hline Index & Expression & Range & Note \\
\hline $\begin{array}{l}\text { Cyclomatic } \\
\text { number }\end{array}$ & $\mu=e-v+G$ & $0 \leq \mu$ & $\begin{array}{l}\text { Number of fundamental circuits } \\
\text { in the network }\end{array}$ \\
\hline Alpha index & $\alpha=\frac{\mu}{2 v-5}$ & $0 \leq \alpha \leq 1$ & $\begin{array}{l}\text { Ratio of number of cycles to } \\
\text { possible maximum number of } \\
\text { cycles }\end{array}$ \\
\hline Beta index & $\beta=\frac{e}{v}$ & $0 \leq \beta$ & $\begin{array}{l}\text { Ratio between number of links } \\
\text { and number of nodes, } \\
\text { equivalent to average degree }\end{array}$ \\
\hline Gamma index & $\gamma=\frac{e}{3(v-2)}$ & $0 \leq \gamma \leq 1$ & $\begin{array}{l}\text { Ratio of number of links to } \\
\text { maximum possible number of } \\
\text { links }\end{array}$ \\
\hline $\begin{array}{l}\text { Average } \\
\text { degree }\end{array}$ & $\bar{d}=\frac{\sum_{i} n_{i}}{v}$ & $\bar{d} \geq 0$ & $\begin{array}{l}\text { Average number of arcs } \\
\text { incident on the nodes }\end{array}$ \\
\hline Cyclicity & $\hat{c}=\frac{\sum_{j=1}^{n} \text { Cycle }_{i}}{|R|}$ & $0 \leq \hat{c} \leq 1$ & $\begin{array}{l}\text { Number of times random walk } \\
\text { led to a cycle back to a } \\
\text { previously visited node/number } \\
\text { of random walks }\end{array}$ \\
\hline \multicolumn{4}{|l|}{ Accessibility } \\
\hline Index & Expression & & Note \\
\hline Diameter & $D=\max \left(d_{i j}\right)$ & \multicolumn{2}{|c|}{$\begin{array}{l}\text { The maximum distance among all shortest } \\
\text { distances between all O-D pairs in the } \\
\text { network }\end{array}$} \\
\hline $\begin{array}{l}\text { Average } \\
\text { Shimbel index }\end{array}$ & $A_{i}=\frac{\sum_{j=1}^{n} d_{i j}}{v-1}$ & \multicolumn{2}{|c|}{$\begin{array}{l}\text { Average of the sum of the lengths of all } \\
\text { shortest paths connecting all pairs of nodes in } \\
\text { the network }\end{array}$} \\
\hline $\begin{array}{l}\text { Betweenness } \\
\text { centrality }\end{array}$ & $B C_{i}=\frac{\sigma_{j k(i)}}{\sigma_{j k}}$ & \multicolumn{2}{|c|}{$\begin{array}{l}\text { Number of times a node is crossed by shortest } \\
\text { paths in the graph }\end{array}$} \\
\hline
\end{tabular}

(Note: $e$ - number of links in the graph, $v$-number of nodes in the graph, $G$-number of sub-graphs in the graph, $n_{i}$-number of arcs incident on node $i, d_{i j^{-}}$distance of shortest path between O-D pair $(i, j), C y c l e_{i^{-}}$ number of times random walk cycled back to node $i,|R|$ - number of random walks, $\sigma_{j k}$-total number of shortest paths from node $j$ to $k, \sigma_{j k(i)}-$ number of shortest paths from node $j$ to $k$ that pass through node i)

In this study, three measures are used to characterize a network: average degree $\bar{d}$, diameter $D$, and cyclicity $\hat{C}$. To obtain a value for cyclicity for a given network topology, a random walk is taken in a large number of randomly generated networks of the same topology 
and size. The random walk is terminated under one of three conditions: (1) n decisions corresponding to the traversal of links, where $\mathrm{n}=1.5^{\cdot} \mathrm{D}$ have been taken, (2) the walk returns to a node that was already visited, and (3) no further move is possible. In each random walk, a link may be traversed at most once. If that link has been used in one direction, it cannot be used again in that direction. For each network, the random walk is attempted 100,000 times. $\hat{C}=$ (number of runs on a network for which the walk returned to a node already visited) / $(100,000 *$ number of nodes in the network). 


\subsection{Defining Resilience}

The term resilience has been used in a variety of ways. In the context of ecological resilience and its relation to social resilience, Adger (2000) summarizes resilience measures as capturing either: (1) the ability of the system to absorb the effects of a disturbance (consistent with Holling's (1973) ecological resilience definition) or (2) the rate of recovery of the system from a disturbance. Herein, a definition described in (Rose, 2004) and mathematically conceptualized in (Chen and Miller-Hooks, 2012; Miller-Hooks et al., 2012; Faturechi and Miller-Hooks, 2013) is adopted. These works define resilience in terms of not only the inherent ability of the system to absorb the effects of the disruption, but also its ability to efficiently adapt post-event.

In these earlier computational works (i.e., Chen and Miller-Hooks, 2012; Miller-Hooks et al., 2012; Faturechi and Miller-Hooks, 2013), the problem of measuring a network's resilience was given as an optimization problem. The problem was formulated as a two-stage stochastic integer program with first-stage preparedness and second-stage recovery decision variables. The stochastic program assumes probabilistic knowledge of a set of potential (chosen or randomly generated) disaster event scenarios. First-stage decisions are taken in preparation for such an event occurrence given: (1) knowledge of the possible scenarios with their impacts and occurrence probabilities and (2) an assessment of system performance under an optimal scenariospecific allocation of fixed monetary and time budgets (i.e. second-stage decisions). Resilience is measured by the ratio of the expected maximum throughput that can be accommodated postdisaster to the required/achievable pre-disaster throughput level given these decisions.

This approach to resilience computation that accounts for the improved performance that can be achieved by taking the optimal post-event adaptive actions under each potential disaster event scenario provides an upper limit on the system's expected resilience for the considered scenarios. Alternative resilience metrics are described in numerous works, a review of which is provided in (Faturechi and Miller-Hooks, 2015). The need for a resilience interpretation including such a 'normative agenda' describing 'to where,' 'to what level' and in what direction' investments are needed in place of a descriptive interpretation is discussed in (Weichselgartner and Kelman, 2014).

Using a similar optimization-based framework, three interpretations of resilience are studied herein. The first is based on throughput using the earlier proposed definition from (Miller-Hooks et al., 2012). The second is based on a concept of connectivity between origin- 
destination pairs, termed $O-D$ connectivity. The third is computed from the average reciprocal distance between all O-D pairs. These interpretations were chosen to incorporate some of the characteristics of classical graph theory measures described in the previous subsection. These measures are consistent with the (1) network attribute-based and (2) connectivity or flow-based classification approaches synopsized in (Grubesic et al., 2008) in relation to network vulnerability. The specific measures applied here, however, account for not only the inherent coping capacity of the network as is key to vulnerability assessment, but also the effects of preparedness actions often included in robustness measurement and the post-disaster adaptability of the system, which is a key factor in resilience quantification.

The original formulation from (Miller-Hooks et al., 2012) with resilience based on throughput and adaptations for $O-D$ connectivity and average reciprocal distance are presented next. Notation used in these formulations are summarized in Table 2, and follow the notation given in Miller-Hooks et al. (2012) where practical.

Table 2 Notation used for problem formulations

\begin{tabular}{|l|l|}
\hline Notation & \multicolumn{1}{c|}{ Description } \\
\hline$W$ & set of O-D pairs of nodes in the network, $W=\left\{w_{i j}, \forall i, j \in N\right\}, N$ is the node set \\
\hline$K_{w}$ & set of paths $k$ connecting O-D pair $w$ \\
\hline$K_{w}^{\mathrm{K}}$ & set of K shortest paths $k$ connecting O-D pair $w$ \\
\hline$D_{w}$ & original demand between O-D pair $w$ \\
\hline$\Gamma_{w}$ & original connectivity of O-D pair $w$ ( $=1$ if connected, $=0$ otherwise) \\
\hline$\Psi_{w}$ & original shortest distance of O-D pair $w$ \\
\hline$R$ & set of available recovery actions $r$ \\
\hline$b_{a r}$ & cost of implementing recovery activity $r R$ on arc $a$ \\
\hline$P$ & set of available preparedness actions $p$ \\
\hline$b_{a p}$ & cost of implementing preparedness activity $p \oplus$ on arc $a$ \\
\hline$b_{a r}^{p}$ & cost of implementing recovery activity $r$ on arc $a$ if preparedness action $p$ is taken \\
\hline$B$ & given budget \\
\hline$M$ & Large integer number \\
\hline$c_{a}(\xi)$ & post-disaster capacity of arc $a$ for disruption scenario $\xi$ \\
\hline$\Delta c_{a p}$ & augmented capacity of arc $a$ given preparedness action p is taken \\
\hline$\Delta c_{a r}(\xi)$ & $\begin{array}{l}\text { augmented capacity of arc } a \text { due to implementing recovery activity } r \text { for disruption } \\
\text { scenario } \xi\end{array}$ \\
\hline
\end{tabular}




\begin{tabular}{|c|c|}
\hline$\Phi_{a}(\xi)$ & post-disaster connectivity of arc $a$ for disruption scenario $\xi$ \\
\hline$\Delta \Phi_{a p}$ & augmented connectivity of arc $a$ given preparedness action $p$ is taken \\
\hline$\Delta \Phi_{a r}(\xi)$ & $\begin{array}{l}\text { augmented connectivity of arc } a,=1 \text { if recovery activity } r \text { is implemented for } \\
\text { disruption scenario } \xi \text {, and }=0 \text { otherwise }\end{array}$ \\
\hline$d_{a}$ & length of arc $a$ \\
\hline$d^{w}(\xi)$ & shortest distance of O-D pair $w$ under disruption $\xi$ \\
\hline$t_{a}(\xi)$ & traversal time of arc $a$ under disruption scenario $\xi$ \\
\hline$t_{a r}$ & traversal time of arc $a$ if recovery activity $r$ is implemented \\
\hline$q_{a r}$ & implementation time of recovery activity $r$ on $\operatorname{arc} a$ \\
\hline$q_{a r}^{p}$ & $\begin{array}{l}\text { traversal time of arc } a \text { if related preparedness action } p \text { and recovery action } r \text { is } \\
\text { implemented }\end{array}$ \\
\hline$Q_{k}^{w}(\xi)$ & maximum implementation time of recovery actions on path $k$ between O-D pair $w$ \\
\hline$T_{\max }^{w}$ & maximum allowed traversal between O-D pair $w$ \\
\hline$\lambda$ & $\begin{array}{l}\text { preparedness-recovery action relationship matrix in which each element } \lambda_{p r} \text { is set to } \\
1 \text { if recovery action } r \text { is affected by preparedness action } p \text { and } 0 \text { otherwise. }\end{array}$ \\
\hline$\delta_{a k}^{w}$ & path-arc incidence ( $=1$ if path $k$ uses arc $a$, and $=0$ otherwise) \\
\hline$\beta_{a p}$ & $\begin{array}{l}\text { binary variable indicating whether or not preparedness activity } p \text { is undertaken on arc } \\
a(=1 \text { if preparedness action } p \text { is taken on arc a and }=0 \text { otherwise })\end{array}$ \\
\hline$\varphi^{w}(\xi)$ & $\begin{array}{l}\text { binary variable indicating whether or not O-D pair } w \text { is connected (=1 if O-D pair } w \\
\text { is connected and }=0 \text {, otherwise) under scenario } \xi\end{array}$ \\
\hline$\varphi_{k}^{w}(\xi)$ & $\begin{array}{l}\text { binary variable indicating whether or not O-D pair } w \text { is connected via path } k(=1 \text { if } \\
\text { path } k \text { is exists and }=0 \text {, otherwise) under scenario } \xi\end{array}$ \\
\hline$X_{a}^{w}(\xi)$ & $\begin{array}{l}\text { binary variable indicating whether or not arc } a \text { is used for O-D pair } w(=1 \text { if link } a \text { is } \\
\text { used and }=0 \text {, otherwise) under scenario } \xi\end{array}$ \\
\hline$y_{k}^{w}(\xi)$ & $\begin{array}{l}\text { binary variable indicating whether or not shipments use path } k(=1 \text { if path } k \text { is used } \\
\text { and }=0 \text { otherwise) between O-D pair } w \text { under scenario } \xi\end{array}$ \\
\hline$f_{k}^{w}(\xi)$ & post-disaster flow of shipments along path $k$ between O-D pair $w$ under scenario $\xi$ \\
\hline$\gamma_{a r}(\xi)$ & $\begin{array}{l}\text { binary variable indicating whether or not recovery activity } r \text { is undertaken on arc } a \text { in } \\
\text { the aftermath of disruption scenario } \xi \text { (=1 if recovery action } r \text { is taken on arc } a \text { and } \\
=0 \text { otherwise) }\end{array}$ \\
\hline
\end{tabular}

For completeness, the resilience formulation of (Miller-Hooks et al., 2012) is presented next. Detailed explanation of the model is given in the earlier work. Modifications to this formulation required for the $O-D$ connectivity and average reciprocal distance variants are provided thereafter. 


$$
\begin{aligned}
& \text { Resilience-Throughput }\left(R^{T}\right) \\
& \max E_{\tilde{\xi}}\left[\max \sum_{w \in W} \sum_{k \in K_{w}} f_{k}^{w}(\xi)\right] / \sum_{w \in W} D_{w}
\end{aligned}
$$

s.t.

$$
\begin{aligned}
& \sum_{p} \beta_{a p} \leq 1, \nabla a \in A \\
& \sum_{k \in K_{w}} f_{k}^{w}(\xi) \leq D_{w}, \quad \forall w \in W \\
& \sum_{a} \sum_{p} b_{a p} \cdot \beta_{a p}+\sum_{a} \sum_{r} b_{a r} \cdot \gamma_{a r}(\xi)+\sum_{a} \sum_{r} \sum_{p}\left(b_{a r}^{p}-b_{a r}\right) \cdot \lambda_{p r} \cdot \beta_{a p} \cdot \gamma_{a r}(\xi) \leq B, \\
& \sum_{w \in W} \sum_{k \in K_{w}} \delta_{a k}^{w} \cdot f_{k}^{w}(\xi) \leq c_{a}(\xi)+\sum_{r} \Delta c_{a p} \cdot \beta_{a p}+\sum_{r} \Delta c_{a r}(\xi) \cdot \gamma_{a r}(\xi), \quad \forall a \in A \\
& \sum_{a \in k} t_{a}(\xi)+\sum_{a \in k} \sum_{r}\left(t_{a r}-t_{a}(\xi)\right) \cdot \gamma_{a r}(\xi)+Q_{k}^{w}(\xi) \leq T_{\max }^{w}+M \cdot\left(1-y_{k}^{w}(\xi)\right), \quad \forall k \in K_{w}, w \in W \\
& f_{k}^{w}(\xi) \leq M y_{k}^{w}(\xi), \quad \forall k \in K_{w}, w \in W \\
& Q_{k}^{w}(\xi)-q_{a r} \cdot \gamma_{a r}(\xi)-\sum_{p}\left(q_{a r}^{p}-q_{a r}\right) \cdot \lambda_{p r} \cdot \beta_{a p} \cdot \gamma_{a r}(\xi) \geq 0, \quad \forall a \in k, k \in K_{w}, w \in W \\
& \sum_{r} \gamma_{a r}(\xi) \leq 1, \forall a \in A, r \in R \\
& \beta_{a p} \in\{0,1\}, \quad \forall a \in A, p \in P \\
& \gamma_{a r}(\xi) \in\{0,1\} \quad \forall a \in A, r \in R \\
& y_{k}^{w}(\xi) \in\{0,1\}, \quad \forall k \in K_{w}, w \in W \\
& f_{k}^{w}(\xi) \text { integer, } \quad \forall k \in K_{w}, w \in W
\end{aligned}
$$

$R^{T}$ is a two-stage stochastic program with binary first-stage variables. The objective (1) seeks the maximum expected throughput that can be accommodated over all disaster scenarios, which are realized in the second stage. The number of shipments that can be accommodated must not exceed the original (pre-disaster) demand for service as guaranteed by Constraints (3). Constraints (4) enforce a limited budget for first- and second-stage actions. Capacity constraints given capacity augmentation due to preparedness or recovery actions are ensured through Constraints (5). Constraints (6)-(8) are level-of-service constraints that guarantee service by $T_{\max }^{w}$ for each O-D pair w. Constraints (2) and (9) limit the number of preparedness or recovery actions that are taken on a given arc to 1 of each type. Note that this is nonlimiting, because multiple actions can be bundled. Binary and integer restrictions are imposed in Constraints (10)-(13) 
In classical connectivity analyses of directed graphs an O-D pair is said to be connected if there is a directed path with positive capacity between the path's origin and its destination. The network is strongly connected if such a path exists between every O-D pair. Consider a set of pre-disaster k-shortest paths for each O-D pair. In $O-D$ connectivity considered herein, an O-D pair is considered connected if one of its pre-disaster k-shortest O-D paths exists, the total connectivity is set as the number of connected O-D pairs, and is, thus, a measure of strong connectivity. Alpha and Gamma indices described in Table 1 capture similar characteristics to this measure. Thus, $K_{w}^{\mathrm{K}}$ is interpreted as the set of pre-disaster k-shortest, loopless paths between O-D pair $w$. Then, resilience with respect to $O-D$ connectivity is formulated as follows.

$$
\begin{aligned}
& \text { Resilience - O-D Connectivity }\left(R^{O D}\right) \\
& \max E_{\tilde{\xi}}\left[\max \sum_{w \in W} \varphi^{w}(\xi)\right] / \sum_{w \in W} \Gamma_{w}
\end{aligned}
$$

s.t.

Constraints (2), (4), (9)-(11)

$$
\begin{aligned}
& \varphi^{w}(\xi) \leq \sum_{k} \varphi_{k}^{w}(\xi), \quad \forall k \in K_{w}^{\mathrm{K}}, w \in W \\
& \varphi_{k}^{w}(\xi) \leq \Phi_{a}(\xi)+\sum_{r} \Delta \Phi_{a p} \cdot \beta_{a p}+\sum_{r} \Delta \Phi_{a r}(\xi) \cdot \gamma_{a r}(\xi), \quad \forall a \in k, k \in K_{w}^{\mathrm{K}}, w \in W \\
& \varphi^{w}(\xi), \varphi_{k}^{w}(\xi) \in\{0,1\}, \quad \forall k \in K_{w}^{\mathrm{K}}, w \in W
\end{aligned}
$$

Objective (14) seeks to maximize the expected proportion of O-D pairs that are connected. Constraints (15) ensure that if an O-D path exists, then each arc along the path must either be in good working order, retrofitted so as to guarantee its operation in any disaster, or repaired post-disaster. Constraints (15) ensure O-D pair $w$ is connected as long as post-disaster there exists one path $k$ of the original $\mathrm{k}$-shortest paths. The connectivity of path $k$ is assessed in constraints (16), where both the post-disaster state of each arc in the path and whether or not it is repaired if damaged are considered. $\varphi^{w}(\xi)$ and $\varphi_{k}^{w}(\xi)$ are restricted to be binary.

Finally, resilience in terms of average reciprocal distance is defined. Average reciprocal distance is similar to the Average Shimbel Index described in Table 1. It is calculated by first computing one over the shortest post-disaster distance of existing paths between each O-D pair $w$. The average reciprocal distance is, thus, computed from the average of these reciprocal 
distances over all O-D pairs. If any O-D pair is disconnected, an exceptionally long distance is associated with that O-D pair. The resilience formulation is revised accordingly next.

Resilience - Average Reciprocal Distance $\left(R^{A R D}\right)$

$\max E_{\tilde{\xi}}\left[\max \sum_{w \in W} \frac{1}{d^{w}(\xi)}\right] / \sum_{w \in W} \frac{1}{\Psi_{w}}$

s.t.

Constraints (2), (4), (9)-(11)

$\sum_{(i, j)=a} X_{a}^{w}(\xi)-\sum_{(j, i)=a} X_{a}^{w}(\xi)=\left\{\begin{array}{l}1 \quad i=O_{w} \\ 0 \quad i \neq O_{w}, L_{w} \\ -1 \quad i=L_{w}\end{array} \quad \forall a \in w, w \in W\right.$
$d^{w}(\xi) \geq \sum_{a} d_{a} X_{a}^{w}(\xi) \quad \forall a \in w, w \in W$
$0 \leq X_{a}^{w}(\xi) \leq \Phi_{a}(\xi)+\sum_{r} \Delta \Phi_{a p} \cdot \beta_{a p}+\sum_{r} \Delta \Phi_{a r}(\xi) \cdot \gamma_{a r}(\xi) \quad \forall a \in w, w \in W$
$X_{a}^{w}(\xi) \in\{0,1\}, d^{w}(\xi)$ integer, $\quad \forall a \in w, w \in W$

Constraints (19) are flow conservation constraints. They ensure that only one path is selected to connect O-D pair $w$. Constraints (20) compute the distance of the O-D path. The objective (18) aims for each such O-D path to be the shortest possible given a fixed budget for repairing damaged links post-disaster. Constraints (21) require that all arcs of each O-D shortest path is functional post-disaster. If an arc of a path does not function, that path will not exist. Binary and integer restrictions on $X_{a}^{w}(\xi)$ and $d^{w}(\xi)$ are indicated in constraints (22).

\subsection{Obtaining Resilience Values: Solution Methodology}

The three models of resilience presented in the previous section are nonlinear as a consequence of bilinear terms (the multiplication of first- and second-stage variables) in the constraints. This nonlinearity can be eliminated by decomposing the problem by stage. Such methods fix the firststage variables before evaluating the second stage. Thus, an exact solution methodology based on such a decomposition method, i.e. integer L-shaped decomposition described in (MillerHooks et al., 2012), is employed herein for solution of formulation $R^{T}$ and was adapted to solve formulations $R^{O D}$ and $R^{A R D}$. The Integer L-shaped method is a variant of Benders' decomposition. 
It was originally proposed in (Laporte and Louveaux 1993) for solving two-stage integer stochastic programs with binary first-stage decision variables. In this method, the two-stage stochastic program is decomposed into a master problem (MP) and set of subproblems (SPs), one for each scenario. In the MP, integrality constraints are relaxed. The MP and SPs are solved iteratively employing a pendant node list used to implement a type of branch-and-bound procedure. Solutions from the SPs provide valid optimality cuts that can be incorporated within the MP in the form of constraints, narrowing the solution space. The solution process terminates when the pendant node list is empty.

This procedure applies directly in solution of $R^{O D}$. As complete enumeration of all paths between all O-D pairs would be computationally intractable for large networks, the k-shortest paths between each O-D pair is generated. This is completed through a preprocessing step. There are several classical algorithms presented in the literature for solving the k-shortest, loopless path problem. A well-known algorithm due to Yen (1971) was implemented for this purpose. The integer L-shaped method is also used to solve $R^{A R D}$. To implement the integer Lshaped method for solution of $R^{T}, R^{O D}$ and $R^{A R D}$, the problem is treated as one of minimization. The reformulation for $R^{T}, R^{O D}$ and $R^{A R D}$ as a MP and set of SPs is given in Table 3.

Table 3 Decomposition for $R^{T}, R^{O D}$ and $R^{A R D}$

\begin{tabular}{|c|c|c|}
\hline & MP & SPs \\
\hline$R^{T}$ & $\begin{array}{l}\min \theta^{T} \\
\text { s.t. Constraints (2) } \\
\sum_{a} \sum_{p} b_{a p} \beta_{a p} \leq B, \\
f\left(\theta^{T}, \beta\right) \geq 0, \quad 0 \leq \beta_{a p} \leq 1\end{array}$ & $\begin{array}{l}E_{\tilde{\xi}}\left[\min \left(-\sum_{w \in W} \sum_{k \in K_{w}} f_{k}^{w}(\xi)\right)\right] \\
\text { s.t. constraints }(6-14)\end{array}$ \\
\hline$R^{O D}$ & $\begin{array}{l}\min \theta^{O D} \\
\text { s.t. Constraints (2) } \\
\qquad \sum_{a} \sum_{p} b_{a p} \beta_{a p} \leq B, \\
f\left(\theta^{O D}, \beta\right) \geq 0, \quad 0 \leq \beta_{a p} \leq 1\end{array}$ & $\begin{array}{l}\qquad E_{\tilde{\xi}}\left[\min \left(-\sum_{w \in W} \varphi^{w}(\xi)\right)\right] \\
\text { s.t. constraint s }(4,9,11,15-17)\end{array}$ \\
\hline
\end{tabular}




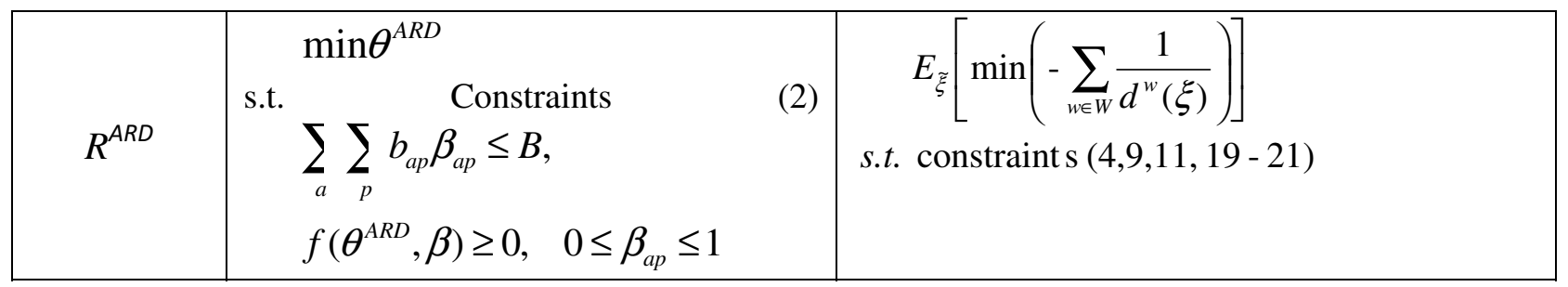

Note that each subproblem in the decomposition of $R^{A R D}$ is an all pairs shortest path problem. Floyd-Warshall's algorithm (Floyd, 1962) can be applied to solve each SP in this case. $D_{w}, \Gamma_{w}, \Psi_{w}$ are constants and need not be considered in the steps of the solution method.

\section{Numerical Experiments}

Numerical experiments were conducted to assess resilience of the network topologies given in Figure 1 through solution of each of the three considered resilience problems. The experiments were completed first on the small (tile-size) networks and then again on their larger extrapolations. All arcs were assumed to have identical pre-disaster capacity. This capacity decreased by $50 \%$ or $100 \%$ if the arc is impacted in a disaster scenario. Arc travel times were assumed to increase $100 \%$ for a capacity drop of 50\%. Arc lengths were set to 1 unit in all networks for all arcs with one exception. Diagonal arcs of the matching pair, complete and diamond networks have lengths consistent with their Euclidean lengths. Regardless of network size, 100 network realizations were considered in scenario generation. In each scenario, the number of impacted arcs, $n$, follows a binomial distribution with parameter $\mathrm{p}=0.25$. $n$ arcs are chosen randomly from a uniform distribution. Half of the chosen arcs had a 50\% drop in capacity and the remaining $50 \%$ had a $90 \%$ drop in capacity. The assignment of the capacity drop is made randomly.

Two preparedness (P1 and P2) and three recovery (R1, R2, and R3) actions are designed to mitigate the impact of disaster. For the $R^{T}$ analysis, the characteristics of these actions are summarized in Table 4. The implementation time and cost of all three recovery actions are reduced by $20 \%$ or $25 \%$ if action $\mathrm{P} 1$ or $\mathrm{P} 2$ is taken for that arc, respectively. 
Table 4 Characteristics of preparedness and recovery actions

\begin{tabular}{ccccc}
\hline Actions & $\begin{array}{c}\text { Recovery activity } \\
\text { duration (units) }\end{array}$ & $\begin{array}{c}\text { Cost } \\
\text { (units) }\end{array}$ & $\begin{array}{c}\text { Increase in arc } \\
\text { capacity (units) }\end{array}$ & $\begin{array}{c}\text { Reduction in arc } \\
\text { traversal time (units) }\end{array}$ \\
\hline P1 & N/A & 10 & 1 & N/A \\
P2 & N/A & 20 & 1 & N/A \\
R1 & 2 & 25 & 2 & 4 \\
R2 & 1 & 10 & 1 & 2 \\
R3 & 3 & 50 & 3 & 5 \\
\hline
\end{tabular}

In the case of $R^{O D}$ and $R^{A R D}$, the three recovery activities restore arc connectivity but at different costs. Though preparedness actions do not directly improve the performance of target arcs in terms of connectivity, in the case of $R^{O D}$ and $R^{A R D}$, such actions can reduce the cost of recovery actions. The cost of recovery and the relationship between preparedness and recovery actions in $R^{O D}$ and $R^{A R D}$ are set to be the same as in $R^{T}$. A budget of 200 and 2,000 units is assumed for small and large networks, respectively.

Table 5 Resilience levels of network topologies (small networks)

\begin{tabular}{|c|c|c|c|c|c|c|c|c|c|c|c|c|c|c|c|}
\hline Type & $v$ & $e$ & $\bar{d}$ & $\mathrm{CC}$ & $\begin{array}{c}\mathrm{P} \\
\text { only }\end{array}$ & $\begin{array}{c}\mathrm{R} \\
\text { only }\end{array}$ & $\begin{array}{c}\mathrm{P} \& \\
\mathrm{R}\end{array}$ & $\mathrm{CC}$ & $\begin{array}{c}\mathrm{P} \\
\text { only }\end{array}$ & $\begin{array}{c}\mathrm{R} \\
\text { only }\end{array}$ & $\begin{array}{c}\mathrm{P} \& \\
\mathrm{R}\end{array}$ & $\mathrm{CC}$ & $\begin{array}{c}\mathrm{P} \\
\text { only }\end{array}$ & $\begin{array}{c}\mathrm{R} \\
\text { only }\end{array}$ & $\begin{array}{c}\mathrm{P} \& \\
\mathrm{R}\end{array}$ \\
\hline 1 & 9 & 12 & 2.6 & 70.6 & 76.2 & 87.3 & 92.2 & 70.4 & 73.2 & 92.4 & 97.3 & 52.1 & 53.3 & 55.2 & 61.7 \\
\hline 2 & 9 & 8 & 1.8 & 65.3 & 72.1 & 78.8 & 85.3 & 70.0 & 78.6 & 83.1 & 92.5 & 44.8 & 46.9 & 51.4 & 58.8 \\
\hline 3 & 9 & 8 & 1.8 & 56.2 & 65.6 & 73.1 & 82.4 & 63.1 & 65.3 & 88.3 & 90.5 & 40.4 & 45.9 & 48.7 & 54.0 \\
\hline 4 & 9 & 9 & 2 & 45.9 & 50.4 & 65.4 & 72.7 & 48.6 & 58.3 & 73.6 & 86.7 & 27.7 & 32.7 & 42.5 & 49.2 \\
\hline 5 & 8 & 16 & 4 & 90.8 & 94.9 & 96.2 & 100 & 92.5 & 98.5 & 100 & 100 & 60.9 & 62.3 & 65.5 & 68.5 \\
\hline 6 & 9 & 16 & 3.6 & 78.1 & 85.2 & 88.6 & 95.3 & 87.9 & 91.2 & 96.5 & 100 & 54.7 & 55.5 & 60.3 & 67.4 \\
\hline 7 & 9 & 36 & 8 & 100 & 100 & 100 & 100 & 100 & 100 & 100 & 100 & 83.5 & 87.3 & 94.5 & 97.3 \\
\hline 8 & 10 & 10 & 2 & 66.6 & 72.5 & 80.8 & 86.4 & 72.4 & 80.2 & 84.7 & 92.8 & 46.1 & 50.2 & 54.8 & 57.8 \\
\hline 9 & 10 & 10 & 2 & 66.3 & 71.9 & 80.5 & 85.3 & 70.5 & 75 & 88.8 & 90.4 & 46.4 & 48.5 & 52.6 & 55.1 \\
\hline 10 & 10 & 10 & 2 & 65.2 & 72.6 & 79.3 & 85.6 & 68.6 & 79.3 & 81.2 & 92.2 & 44.1 & 49.7 & 52.2 & 57.3 \\
\hline 11 & 10 & 9 & 1.8 & 52.5 & 57.2 & 72.6 & 79.3 & 57.2 & 60.4 & 77.8 & 81.1 & 29.6 & 36.6 & 44.2 & 52.3 \\
\hline 12 & 9 & 12 & 2.6 & 75.4 & 81.7 & 88.3 & 94.3 & 84.6 & 85.6 & 97.4 & 98.5 & 54.4 & 56.3 & 58.7 & 65.7 \\
\hline 13 & 9 & 8 & 1.8 & 61.0 & 69.3 & 72.6 & 80.2 & 61.5 & 71.6 & 75.6 & 86.1 & 45.2 & 48.7 & 51.9 & 58.9 \\
\hline 14 & 9 & 10 & 2.2 & 65.8 & 76.3 & 82.4 & 91.6 & 71.9 & 80 & 92.2 & 93.3 & 46 & 50.8 & 56.7 & 61.4 \\
\hline 15 & 9 & 12 & 2.6 & 64.3 & 69.3 & 77.4 & 85.4 & 68.4 & 70.4 & 79.8 & 89.9 & 45.2 & 48.9 & 53.7 & 57.2 \\
\hline 16 & 9 & 12 & 2.6 & 62.2 & 67.5 & 73.6 & 82.6 & 65.4 & 68.1 & 76.1 & 86.6 & 42.1 & 44.5 & 47.6 & 53.8 \\
\hline 17 & 9 & 12 & 2.6 & 60.3 & 66.2 & 70.7 & 76.1 & 64.7 & 69.2 & 72.5 & 78.0 & 41.3 & 44.0 & 47.2 & 52.1 \\
\hline
\end{tabular}

* 1 Grid, 2 Hub-and-Spoke, 3 Double Tree, 4 Ring Network, 5 Matching Pairs, 6 Complete grid network, 7 Complete network, 8 Central ring, 9 Double-U, 10 Converging tails, 11 Diverging tails, 12 Diamond Network, 13 Crossing paths networks, 14 single depot network, 15 Random network, 16 Scale-free, 17 Small-world

$* \bar{d}$ Average degree of network 
For each resilience measure, results are given in Tables 5 and 6 terms of: coping capacity (CC), preparedness only $(\mathrm{P})$, recovery only $(\mathrm{R})$, and preparedness and recovery (P \& R). These implementations can be controlled by forcing the preparedness and recovery action decision variables to zero as appropriate, or eliminating or limiting the budget to only a subset of action types. For example, if the budget were eliminated, the system's inherent coping capacity would be measured.

Table 6 Resilience levels of network topologies (large networks with 100 nodes)

\begin{tabular}{|c|c|c|c|c|c|c|c|c|c|c|c|c|c|c|c|}
\hline \multirow[b]{2}{*}{ Type } & \multirow[b]{2}{*}{$\bar{d}$} & \multirow[b]{2}{*}{$D$} & \multirow[b]{2}{*}{$\widehat{C}$} & & \multicolumn{4}{|c|}{$K$} & \multicolumn{4}{|c|}{$R^{A R D}$} \\
\hline & & & & $\mathrm{CC}$ & $\begin{array}{c}\mathrm{P} \\
\text { only }\end{array}$ & $\begin{array}{c}\mathrm{R} \\
\text { only }\end{array}$ & $\begin{array}{c}\mathrm{P} \& \\
\mathrm{R}\end{array}$ & $\mathrm{CC}$ & $\begin{array}{c}\mathrm{P} \\
\text { only }\end{array}$ & $\begin{array}{c}\mathrm{R} \\
\text { only }\end{array}$ & $\begin{array}{c}\mathrm{P} \& \\
\mathrm{R}\end{array}$ & $\mathrm{CC}$ & $\begin{array}{c}\mathrm{P} \\
\text { only }\end{array}$ & $\begin{array}{c}\mathrm{R} \\
\text { only }\end{array}$ & $\begin{array}{c}\mathrm{P} \& \\
\mathrm{R}\end{array}$ \\
\hline 1 & 3.6 & 18 & 67 & .6 & 79.5 & 87.3 & 92.2 & 71.9 & 76.3 & 91 & 96.3 & 53.9 & 55.8 & 58.6 & 64.9 \\
\hline 2 & & & & 1 & & 1.5 & 90.3 & 1.3 & .3 & .6 & .2 & 6.3 & 1.3 & 5.8 & 3.8 \\
\hline 3 & & & & & & 5.1 & 82.7 & 64. & 4.3 & & .6 & 41.8 & & 52.2 & \\
\hline 4 & 2 & & & & & 2.4 & 75.6 & 53.8 & 4 & J & .1 & 53 & & 46.0 & 3.1 \\
\hline 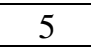 & & & & 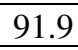 & & 97.4 & 100 & 94.0 & 95 . & 11 & 100 & 62.8 & 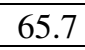 & .7 & 0.7 \\
\hline 6 & 84 & & 31.0 & 79.2 & 88 & 92.3 & 97.4 & 91.1 & 92.4 & 95.6 & 100 & 58.1 & 2 & 64.1 & 69.5 \\
\hline 7 & 99 & 1 & 100 & 100 & 100 & 100 & 100 & 100 & 100 & 100 & 100 & 88.2 & 9.2 & 95.9 & 98.9 \\
\hline 8 & 2 & & & 67.6 & 76.1 & 81.4 & 91.1 & 74.1 & 82.8 & 88.2 & 94.8 & 48.1 & 54.6 & 58.4 & 64.2 \\
\hline 9 & 2 & 4 & & 70.5 & 74.6 & 82.7 & 89.3 & 72.5 & 75.4 & 90.0 & 95.0 & 51.1 & 51.2 & 55.1 & 58.3 \\
\hline 10 & 2 & & & 69.5 & 74.6 & 81.7 & 89.2 & 76.1 & 82.3 & 82.8 & 93.9 & 47.0 & 3 & 55.1 & 60.3 \\
\hline 11 & (20 & 6 & & 53.2 & 57.6 & 73.1 & 84.9 & 58.9 & 63.0 & 77.8 & 82.7 & 31.4 & 39.2 & 44.7 & 54.4 \\
\hline 12 & 4 & 5 & & 76.7 & 85.4 & 91.1 & 96.1 & 88.0 & 89.1 & 94.6 & 97.2 & 58.0 & 61.4 & 62.7 & 67.9 \\
\hline 13 & 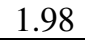 & 5 & & 63.3 & 71.7 & 74.1 & 85.3 & 64.0 & 71.6 & 76.5 & 91.2 & 48.6 & 1 & 53.6 & 60.9 \\
\hline 14 & 2.02 & 45 & & 67.1 & 76.8 & 82.8 & 93.2 & 73.1 & 80.3 & 94.9 & 95.7 & 47.8 & 3 & 59.2 & 64.9 \\
\hline 15 & 3.6 & 18 & 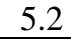 & 69.2 & 76.5 & 81.0 & 87.3 & 72.2 & 77.8 & 83.0 & 91.5 & 46.0 & 99.9 & 54.5 & 57.4 \\
\hline 16 & 3.6 & - & 7.1 & 65.8 & 69.3 & 74.4 & 82.9 & 69.1 & 72.8 & 76.3 & 84.3 & 42.1 & 45.3 & 48.4 & 54.3 \\
\hline 17 & 3.6 & 18 & 3.8 & 64.6 & 68.1 & 72.3 & 80.4 & 66.2 & 70.3 & 75.4 & 81.6 & 41.4 & 44.7 & 47.6 & 52.9 \\
\hline
\end{tabular}

* 1 Grid, 2 Hub-and-Spoke, 3 Double Tree, 4 Ring Network, 5 Matching Pairs, 6 Complete grid network, 7 Complete network, 8 Central ring, 9 Double-U, 10 Converging tails, 11 Diverging tails, 12 Diamond Network, 13 Crossing paths networks, 14 single depot network, 15 Random network, 16 Scale-free, 17 Small-world $* 2 \bar{d}$ - average degree, $L$ - diameter, and $\hat{C}$ - cyclicity.

The analysis directly applies to the system-level resilience concept that treats each transportation component as simple nodes with no properties or specific structure. These components, however, can be complex networks in their own right. Thus, it was assumed that an event that impacts a component's capacity will be realized through a reduction in capacity of incident links. Consequently, additional experiments were conducted to explore the relationship between component health and system resilience. In these experiments, components are defined as a single tile of the network or by selecting a small portion of the network. When a component is impacted by disaster, only the constituent nodes and arcs will incur capacity reductions. A related concept is employed by Jenelius et al. (2012) wherein a region containing the Swedish 
roadway network was divided into uniformly shaped cells of up to $50 \mathrm{~km}$ by $50 \mathrm{~km}$. Their goal, however, was to evaluate cell importance in spatially spread disruption events.

For small networks, damage to a single randomly selected arc or node (.5 probability of each) was simulated. 100 such simulations were run. In the runs with damage to a node, capacity of all incident arcs (incident on or emanating from the node) was reduced by either 50\% or $100 \%$ (.5 probability of each). A similar capacity reduction pattern was employed for damage to a single arc. In each simulation run on the large networks, damage was imposed on one randomly chosen "tile" used in creating the larger network structure. The capacity of all arcs within the damaged tile was reduced by between 50 and 100\% (.5 probability of each). 100 such simulations were run in this experiment.

Table 7 Results of system health (small networks)

\begin{tabular}{|c|c|c|c|c|c|c|c|c|c|c|c|c|c|c|c|}
\hline \multirow{2}{*}{ Type } & $v$ & $e$ & $\bar{d}$ & \multicolumn{4}{|c|}{ CC in $R^{T}$} & \multicolumn{4}{|c|}{ CC in $R^{O D}$} & \multicolumn{4}{|c|}{ CC in $R^{\text {ARD }}$} \\
\hline 1 & 9 & 12 & 2.6 & 80.6 & 87.0 & 74.5 & 4.5 & 82.6 & 89.4 & 77.9 & 2.6 & 54.2 & 60.1 & 48.2 & 2.7 \\
\hline 2 & 9 & 8 & 1.8 & 76.0 & 81.9 & 69.1 & 4.6 & 77.6 & 83.8 & 72.3 & 3.6 & 45.1 & 51.6 & 38.6 & 3.7 \\
\hline 3 & 9 & 8 & 1.8 & 69.5 & 73.8 & 63.0 & 5.2 & 73.7 & 76.3 & 67.9 & 3.6 & 42.1 & 49.0 & 37.9 & 2.9 \\
\hline 4 & 9 & 9 & 2 & 58.8 & 64.5 & 54.8 & 3.3 & 62.3 & 68.2 & 56.1 & 2.0 & 31.6 & 39.0 & 23.9 & 3.8 \\
\hline 5 & 8 & 16 & 4 & 91.6 & 99.6 & 88.2 & 6.4 & 92.4 & 98.6 & 88.8 & 5.2 & 55.5 & 58.7 & 49.1 & 2.3 \\
\hline 6 & 9 & 16 & 3.6 & 88.7 & 95.0 & 83.5 & 6.8 & 89.3 & 95.5 & 84.4 & 5.0 & 53.1 & 58.5 & 47.2 & 1.9 \\
\hline 7 & 9 & 36 & 8 & 100 & 100 & 100 & 0.0 & 100 & 100 & 100 & 0.0 & 90.1 & 92.2 & 87.6 & 0.8 \\
\hline 8 & 10 & 10 & 2 & 74.7 & 81.3 & 66.1 & 5.4 & 76.8 & 85.9 & 69.1 & 4.7 & 46.2 & 54.3 & 36.4 & 4.4 \\
\hline 9 & 10 & 10 & 2 & 74.8 & 85.3 & 70.2 & 5.4 & 76.3 & 85.9 & 68.3 & 3.7 & 45.6 & 56.1 & 38.5 & 4.8 \\
\hline 10 & 10 & 10 & 2 & 74.2 & 88.3 & 70.9 & 6.0 & 77.9 & 86.2 & 73.5 & 5.0 & 45.0 & 56.4 & 40.3 & 3.6 \\
\hline 11 & 10 & 9 & 1.8 & 67.2 & 70.5 & 64.2 & 2.8 & 72.0 & 74.5 & 67.8 & 0.9 & 31.3 & 37.1 & 29.9 & 2.0 \\
\hline 12 & 9 & 12 & 2.6 & 85.1 & 87.9 & 76.3 & 4.4 & 87.1 & 92.4 & 72.5 & 2.5 & 54.2 & 58.2 & 43.0 & 4.4 \\
\hline 13 & 9 & 8 & 1.8 & 69.6 & 74.0 & 64.1 & 4.3 & 71.0 & 78.1 & 65.7 & 2.6 & 45.8 & 51.4 & 38.6 & 3.5 \\
\hline 14 & 9 & 10 & 2.2 & 78.9 & 80.1 & 70.2 & 5.4 & 81.5 & 87.5 & 73.4 & 3.4 & 49.0 & 52.0 & 39.5 & 3.0 \\
\hline 15 & 9 & 12 & 2.6 & 78.0 & 87.5 & 70.4 & 6.3 & 79.1 & 86.2 & 73.6 & 4.2 & 50.4 & 58.7 & 42.3 & 5.7 \\
\hline 16 & 9 & 12 & 2.6 & 75.2 & 83.3 & 68.2 & 7.6 & 77.3 & 84.4 & 68.5 & 6.9 & 46.3 & 53.2 & 40.9 & 6.3 \\
\hline 17 & 9 & 12 & 2.6 & 72.5 & 80.1 & 65.3 & 7.2 & 75.6 & 80.9 & 67.3 & 5.5 & 45.6 & 54.8 & 39.5 & 5.2 \\
\hline
\end{tabular}

* 1 Grid, 2 Hub-and-Spoke, 3 Double Tree, 4 Ring Network, 5 Matching Pairs, 6 Complete grid network, 7 Complete network, 8 Central ring, 9 Double-U, 10 Converging tails, 11 Diverging tails, 12 Diamond Network, 13 Crossing paths networks, 14 single depot network, 15 Random network, 16 Scale-free, 17 Small-world * $\bar{R}$-average resilience level, Max-Maximum resilience level, Min-Minimum resilience level, $s$-stand deviation of resilience

Resilience estimates from these runs involving component-based damage are provided in Tables 7 and 8. Note that only the coping capacity (i.e. in which no preparedness or recovery actions are considered) is considered in measures reported in Table 7, because any action could restore full system capacity due to the experimental setting. These small-network experiments were intended to provide insight into the inherent capabilities of each of the generic topological 
structures to withstand damage. On the larger networks, the network effects of post-damage repair actions were studied through comparison of the resulting resilience measures (i.e. CC, P, R and $P \& R$ ) in Table 8 . This resilience measure that captures component-level effects on systemlevel resilience allows decision makers to assess the magnitude of the benefits that can be derived from the application of security and recovery measures that target individual components or subsystems, including technology implementations and changes to the physical infrastructure.

Table 8 Results of system health (100-node networks)

\begin{tabular}{|c|c|c|c|c|c|c|c|c|c|c|c|c|c|c|c}
\hline \multirow{2}{*}{ Type } & $\bar{d}$ & $D$ & $\hat{C}$ & $\mathrm{CC}$ & $\begin{array}{c}\mathrm{P} \\
\text { only }\end{array}$ & $\begin{array}{c}\mathrm{R} \\
\text { only }\end{array}$ & $\begin{array}{c}\mathrm{P} \& \\
\mathrm{R}\end{array}$ & $\mathrm{CC}$ & $\begin{array}{c}\mathrm{P} \\
\text { only }\end{array}$ & $\begin{array}{c}\mathrm{R} \\
\text { only }\end{array}$ & $\begin{array}{c}\mathrm{P} \& \\
\mathrm{R}\end{array}$ & $\mathrm{CC}$ & $\begin{array}{c}\mathrm{P} \\
\text { only }\end{array}$ & $\begin{array}{c}\mathrm{R} \\
\text { only }\end{array}$ & $\begin{array}{c}\text { P\& } \\
\mathrm{R}\end{array}$ \\
\hline 1 & 3.6 & 18 & 6.7 & 82.5 & 90.0 & 94.8 & 98.3 & 82.8 & 90.8 & 95.7 & 98.5 & 56.6 & 59.2 & 61.6 & 68.0 \\
\hline 2 & 1.98 & 20 & 0.0 & 77.0 & 85.3 & 92.1 & 96.7 & 78.1 & 85.7 & 96.0 & 97.4 & 50.2 & 56.4 & 60.2 & 66.2 \\
\hline 3 & 1.98 & 24 & 0.0 & 73.6 & 78.0 & 84.1 & 88.5 & 74.1 & 76.1 & 85.4 & 89.2 & 44.8 & 52.9 & 56.4 & 62.4 \\
\hline 4 & 2 & 50 & 0.0 & 62.1 & 69.6 & 77.3 & 82.3 & 62.2 & 70.7 & 78.3 & 83.5 & 37.2 & 42.2 & 50.4 & 57.7 \\
\hline 5 & 50 & 51 & 67.6 & 95.0 & 98.6 & 100 & 100 & 95.8 & 99.6 & 100 & 100 & 64.9 & 69.0 & 69.4 & 73.4 \\
\hline 6 & 6.84 & 13 & 31.0 & 91.9 & 95.3 & 99.2 & 100 & 93.1 & 96.3 & 99.6 & 100 & 61.3 & 63.0 & 67.0 & 72.8 \\
\hline 7 & 99 & 1 & 100 & 100 & 100 & 100 & 100 & 100 & 100 & 100 & 100 & 96.5 & 98.7 & 100 & 100 \\
\hline 8 & 2 & 23 & 0.0 & 76.7 & 81.8 & 88.8 & 94.3 & 77.4 & 82.1 & 89.3 & 94.7 & 51.3 & 59.1 & 62.1 & 65.2 \\
\hline 9 & 2 & 48 & 0.2 & 76.6 & 84.3 & 88.7 & 93.6 & 77.0 & 85.3 & 90.0 & 94.1 & 54.3 & 55.9 & 59.3 & 62.4 \\
\hline 10 & 2 & 97 & 0.0 & 75.8 & 82.4 & 88.9 & 95.8 & 77.1 & 83.1 & 89.3 & 96.2 & 50.3 & 57.3 & 58.4 & 62.3 \\
\hline 11 & 1.98 & 66 & 0.0 & 67.5 & 76.3 & 85.3 & 90.4 & 67.9 & 76.4 & 86.3 & 90.5 & 33.2 & 41.9 & 47.1 & 56.7 \\
\hline 12 & 4 & 51 & 0.0 & 87.9 & 92.8 & 95.8 & 100 & 89.3 & 93.4 & 96.9 & 100 & 61.8 & 66.5 & 66.8 & 72.3 \\
\hline 13 & 1.98 & 51 & 0.0 & 71.6 & 73.6 & 82.7 & 86.9 & 72.4 & 74.2 & 82.7 & 87.6 & 51.0 & 54.5 & 56.1 & 63.7 \\
\hline 14 & 2.02 & 49 & 0.0 & 79.6 & 85.1 & 92.2 & 97.5 & 79.8 & 85.8 & 93.3 & 97.8 & 50.1 & 55.8 & 62.4 & 67.9 \\
\hline 15 & 3.6 & 18 & 5.2 & 78.4 & 87.5 & 90.2 & 96.5 & 79.2 & 88.1 & 91.3 & 96.8 & 53.2 & 57.3 & 59.2 & 63.1 \\
\hline 16 & 3.6 & 18 & 4.1 & 75.8 & 81.6 & 86.2 & 91.3 & 76.7 & 83.2 & 86.8 & 92.3 & 50.3 & 52.6 & 55.4 & 60.5 \\
\hline 17 & 3.6 & 18 & 3.8 & 73.6 & 80.3 & 84.7 & 89.6 & 74.6 & 81.4 & 86.2 & 91.7 & 49.6 & 51.9 & 54.4 & 58.8 \\
\hline
\end{tabular}

* 1 Grid, 2 Hub-and-Spoke, 3 Double Tree, 4 Ring Network, 5 Matching Pairs, 6 Complete grid network, 7 Complete network, 8 Central ring, 9 Double-U, 10 Converging tails, 11 Diverging tails, 12 Diamond Network, 13 Crossing paths networks, 14 single depot network, 15 Random network, 16 Scale-free, 17 Small-world

Statistical analyses were conducted on the large networks to investigate the existence of correlation between coping capacity with respect to $R^{T}, R^{O D}$ and $R^{A R D}$ of a topology and network structure as characterized by average degree $\bar{d}$, diameter $L$, and cyclicity $\hat{C}$. Results of correlation analyses are given in Table 9. The results indicate that resilience level is relatively strongly correlated with average degree. Cyclicity is also positively correlated with resilience, but the correlation is less significant as compared with average degree. Diameter is negatively, although weakly, correlated with resilience. All metrics $(\bar{d}, L, \widehat{C})$ are most strongly correlated with $R^{T}$ of the three resilience measures. 
Table 9 Correlation of coping capacity of resilience and $\bar{d}, L$, and $\bar{C}$ in large network

\begin{tabular}{|c|c|c|c|}
\hline & $R^{T}$ & $R^{O D}$ & $R^{A R D}$ \\
\hline Average degree $(\bar{d})^{T}$ & 0.79 & 0.70 & 0.65 \\
\hline Diameter $(\mathcal{L})$ & -0.28 & -0.32 & -0.21 \\
\hline Cyclicity $(\bar{C})$ & 0.83 & 0.73 & 0.71 \\
\hline
\end{tabular}

The difference between resilience with only single-component damage and resilience with damage imposed on a randomly selected arcs is given in Table 10. The results indicate that throughput is most vulnerable to component-level damage. Overall, damage that is concentrated in a small portion of the network is more damaging in terms of resiliency than when the same total damage level is randomly spread about the network.

Table 10 Difference between component health and overall system resilience

\begin{tabular}{|c|c|c|c|}
\hline & $R^{T}$ & $R^{O D}$ & $R^{A R D}$ \\
\hline $\mathrm{CC}$ & 7.23 & 6.18 & 4.12 \\
\hline $\mathrm{P}$ & 6.25 & 7.52 & 3.17 \\
\hline $\mathrm{R}$ & 5.74 & 4.96 & 2.41 \\
\hline $\mathrm{P} \& \mathrm{R}$ & 3.85 & 3.44 & 2.32 \\
\hline
\end{tabular}

Additionally, regression models with dependent variables $R^{T}, R^{O D}$ and $R^{A R D}$ and explanatory variables $\bar{d}, L$, and $\bar{C}$ were estimated. These models and measures of goodness-offit in terms of R-square and Significance F are provided in Table 11 . The closer to 1.0 the Rsquare value, the better the fit. A Significance F of less than 0.05 indicates a good fit at a $95 \%$ confidence interval. It can be noted that with only 17 data points, the R-square values in some cases are poor; thus, only limited insights from the equations should be drawn. Experimentation with additional model forms, e.g. nonlinear forms, may produce betting fitting models. Results from statistical analysis, however, indicate with $95 \%$ confidence that all three graph theory metrics are mostly significant in all equations.

It is interesting to note that while the coefficient of $\hat{C}$ is always positive, the coefficients of $\bar{d}$ and $D$ vary between positive and negative values. Compared with $\bar{d}$ and $D, \widehat{C}$ was found to have greater impact on network resilience. In the case of $\bar{d}$, the coefficient is negative for all implementations of $R^{T}$ and $R^{O D}$, but is positive for all implementations of $R^{A R D}$. In the case of $D$, however, there is no discernible pattern. This change in sign implies possible correlation between these variables. Thus, these equations can be used to provide quick, rough estimates of system- 
level resilience for the various resilience measures and types of investment strategies (e.g. no investment, preparedness only, recovery only, both preparedness and recovery).

Table 11 Estimated resilience regression equations

\begin{tabular}{|c|c|c|}
\hline Estimated regression equation & R-square & Significance F \\
\hline$R^{T}(C C)=64.63-0.16 \bar{d}-0.002 D+0.51 \widehat{C}$ & 0.70 & 0.005 \\
\hline$R^{T}(P)=73.13-0.26 \bar{d}-0.038 D+0.53 \widehat{C}$ & 0.56 & 0.011 \\
\hline$R^{T}(R)=78.14-0.26 \overline{\bar{d}}+0.013 \mathrm{D}+0.47 \bar{C}$ & 0.55 & 0.013 \\
\hline$R^{T}(P \& R)=86.08-0.22 \bar{d}+0.031 D+0.35 \widehat{C}$ & 0.45 & 0.040 \\
\hline$R^{O D}(C C)=69.04-0.36 \bar{d}+0.02 D+0.65 \widehat{C}$ & 0.63 & 0.003 \\
\hline$R^{O D}(P)=72.9-0.31 \bar{d}+0.041 D+0.57 \widehat{C}$ & 0.58 & 0.008 \\
\hline$R^{O D}(R)=82.48-0.246 \bar{d}+0.029 D+0.42 \hat{C}$ & 0.45 & 0.045 \\
\hline$R^{O D}(P \& R)=89.7-0.21 \bar{d}+\underline{\underline{0.028}} D+0.31 \hat{C}$ & 0.34 & 0.134 \\
\hline$R^{A R D}(C C)=46.68+0.19 \bar{d}-0.048 D+0.19 \widehat{C}$ & 0.75 & 0.001 \\
\hline$R^{A R D}(P)=49.96+0.26 \bar{d} \underline{\underline{-0.025}} D+0.12 \widehat{C}$ & 0.74 & 0.001 \\
\hline$R^{A R D}(R)=54.68+0.32 \bar{d}-0.04 D+0.058 \widehat{C}$ & 0.79 & 0.002 \\
\hline$R^{A R D}(P \& R)=59.2+0.32 \bar{d}-0.005 D+0.05 \hat{C}$ & 0.76 & 0.001 \\
\hline
\end{tabular}

Note: double underline indicates that the parameter is statistically insignificant

\section{Analysis of Results}

Analysis of the results from the larger networks (with 100 nodes) given in the prior section provides several important insights. In general, throughput, O-D connectivity and average reciprocal distance resilience measures increase with average degree and greater cyclicity, but decrease with network diameter. Thus, as one would expect, the complete network has the highest values of resilience, while the ring network has the lowest. Additional analyses provide deeper insights into topological-performance relationships. In all network topologies, improvements in all types of resilience are obtained from taking preparedness and/or recovery actions. The highest level is attained when both preparedness and recovery options are allowed. The improvement gains from recovery actions are more significant than from preparedness actions. The significance of these actions appears to be greatest for those networks with the lowest coping capacities. The overall ordering of the network topologies from most resilient to least resilient was found to be: complete, matching pairs, complete grid, diamond, grid, single depot, central ring, hub-and-spoke, double-u, converging tails, random, scale-free, small-world, crossing path, double tree, diverging tails and ring network. This ordering indicates a strong connection between resilience and average degree. 
Generally, networks with higher coping capacity also have higher resilience level (accounting for the implementation of preparedness and recovery actions). Rankings under each category are similar, with change in ranking only for double tree and diamond networks for resilience with preparedness only. With only one exception for the hub-and-spoke network, resilience in terms of $O-D$ connectivity is always higher than resilience in terms of throughput, which is always higher than resilience in terms of average reciprocal distance.

The studied network topologies might also be categorized by type of connections, specifically: group 1 (highly connected) - grid, matching pair, complete grid and diamond networks; group 2 (centrally connected) - hub-and-spoke, double tree, ring, diverging tails and crossing paths networks; group 3 (circuit-like connected) - central ring, double U and converging tails; group 4 (randomly connected) - random, scale-free and small-world networks. Group 1 networks are more often found in urban street systems of larger cities. Group 2 networks are most commonly adopted in air networks. Group 3 networks have wide application in underground transit systems as well as many urban roadway systems. Both urban transit networks and intercity roadway systems can be well represented by Group 4 networks.

Experimental results indicate that those networks falling in group 2 were the least resilient; however, they were also the most responsive to post-event response actions. Of particular note, the ring, diverging tails and crossing paths networks of this group have poor inherent coping capacities but the greatest gain in performance when response actions are incorporated. This implies that for air and other networks with such structure, disaster preparedness is most critical. Networks in group 4 were found to be the second to least resilient of the network groups. Their randomly developed characteristics result in sparsely connected subnetworks. However, in comparison to group 2 networks, group 4 networks may have multiple such hubs (i.e. nodes with high average degree), providing some level of redundancy and opportunities for achieving benefits of potential response actions. This might imply that for air networks, the addition of secondary or tertiary hubs could lead to improved overall resiliency. This can be weighed against the added cost of operating multiple hubs.

Additional insights can be gleaned from comparing the second best performing network group, group 3, to the worst performing group, group 2. Networks in group 2 are connected through a center point, which if damaged will disconnect the network. In topologies with circuit structures as in group 3, there is a redundant connection as failure of a single link is insufficient 
to cause such disconnectivity. The benefit of the circular line structure was also noted by Rodríguez-Núñez and García-Palomares (2014) who studied the vulnerability of the metro system in Madrid. This has implications for the design of new underground rail lines when considering not only coverage of new service areas, but also system effects of such decisions. Thus, steering away from a hub-based design to one with circuits could provide significant improvements in system resilience.

As opposed to network topologies in groups 2 and 3 in which most O-D pairs are connected by only one or two paths, more than five paths were found connecting all O-D pairs in group 1 networks. Thus, these networks experienced the highest resilience levels.

In a final comparison of groups, that group 3 outperformed group 4 networks indicates that, as opposed to making myopic expansion decisions, regardless of mode, long-term, strategic planning for the design of our transportation networks can have significant impact on system resiliency. More generally, to enhance network resilience, investment policies might be devised to strategically transform a network structure falling within a less resilient network group to one with greater resilience through careful addition of redundant links, central connections, secondary or tertiary hubs or similar. Moreover, since such expansion activities are costly and disaster events arise with low-probability, it is important to take into account the additional coping capacity that can be derived through post-event response actions in choosing among the many promising investment options.

Other insights were gleaned from this analysis. For a comparable level of disasterinduced damage in networks with similar average degree, networks with critical arcs (i.e. arcs whose removal will cause the network to be disconnected) tend to be less resilient in terms of all three resilience measures considered herein despite that those arcs were not specifically targeted in the experiments. Such critical arcs are especially prevalent in double tree, diverging tail and crossing path networks. Greater percentage increase in resilience level was observed in such networks when preparedness and recovery actions were implemented than when similar actions were taken to rectify or mitigate damage in other network classes. Furthermore, the benefits derived from taking resilience enhancing actions are greatest for networks containing the greatest number of such critical arcs.

Networks with higher diameter are often sparser and contain less redundant connections. Consequently, they are more vulnerable and less resilient to disaster. For example, the double-U 
network with smaller diameter is more resilient than the diverging tails network. Given comparable average degree and diameter, networks with higher cyclicity tend to be more resilient. Cycles by definition contain redundancy in that the removal of a single arc will not cause a loss in connectivity. This is exemplified by comparing the grid and random networks, where grid networks have higher cyclicity and also notably greater resilience values.

The tested random, scale-free and small-world networks have the same average degree as the grid network, however, these networks tended to be less resilient (Table 8). Moreover, scalefree and small-world networks were found to be less resilient than random networks. This may be because scale-free and small-world networks include nodes with comparatively extreme (high and low) degrees. Thus, some portions of these networks are highly redundant while others are more vulnerable to single-link failure.

In considering the relationship between component health and system health, one can see (Table 8) that the resilience level of the ring and converging trail networks is most affected of all network groups by degradation in the health of a system component. From the statistical analysis, it can be generally concluded that the average degree and cyclicity are better indicators of resiliency than diameter regardless of resilience measure.

Finally, this study provides insights into which network topologies perform best given potential network-development goals and the possibility of taking post-event response actions. Such network topologies may be considered weak if their post-event adaptability is excluded from resilience measurement. Consider the hub-and-spoke network. This network appears poor in comparison to the random network when considering both $R^{T}$ and $R^{O D}$ resilience measures. However, the same network outperforms the random network when preparedness and recovery actions are incorporated in these resilience measurements. Random and scale-free networks were found to outperform crossing paths networks when only the coping capacity is considered; however, when incorporating adaptability within the performance metric, the comparative performance was reversed. That is, the crossing paths networks outperformed the random and scale-free networks under all resilience measures when these post-event actions are included. Another reversal in performance superiority is noted in comparing double tree and scale-free networks when post-event adaptability is incorporated in resilience measurement. 


\section{Conclusions and Extensions}

There are several general conclusions that can be drawn from the results of the numerical experiments. Specifically, the more redundancies built into the network, as indicated by average degree and cyclicity metrics, the greater the resilience level. These insights have implications for transportation applications. For example, in designing and expanding transit systems, it may be desirable to add new services to create loops. This is especially desirable within a central business district where origin-destination patterns may be more uniformly distributed over space. Consider that such a transformation would effectively convert a crossing path network to a central ring network, thus creating a more resilient overall structure. The extension to central ring network provides riders with more transfer opportunities and leads to more redundant network designs.

Studying the differences in resilience of the various network topologies can provide a deeper understanding of how the addition or subtraction of specific links can affect system performance. This can have implications for transportation network planning, as well as disaster preparedness and response.

The ability to compute such a resilience index allows decision makers to assess the potential impact of greater investment levels for recovery actions on facility resilience, as well as the magnitude of the benefits that can be derived from the application of security measures, including technology implementations and changes to the physical infrastructure.

Findings presented here are limited by the tested resilience measures, origin-destination demand pattern assumptions, and other aspects of the experimental settings. One might consider alternative measures and experiments to investigate additional properties of these network topologies.

Acknowledgments: This work was funded by the National Science Foundation. This support is gratefully acknowledged, but implies no endorsement of the findings.

\section{References}

1. Adger, W., 2000. Social and ecological resilience: are they related? Progress in Human Geography 24 (3), 347-364. 
2. Barabási, A.L., Albert, R., 1999. Emergence of scaling in random networks. Science 286,509-12.

3. Chen, L., Miller-Hooks, E., 2012. Resilience: An indicator of recovery capability in intermodal freight transport. Transportation Science 46, 109-123.

4. Derrible, S., Kennedy, C., 2010. The complexity and robustness of metro networks. Physica A 389, 3678-3691.

5. Faturechi, R., Miller-Hooks, E., 2015. Measuring performance of transportation infrastructure systems in disaster: a comprehensive review. In press, ASCE Journal of Infrastructure Systems 21(1), pages to be announced.

6. Floyd, R.W., 1962. Algorithm 97, Shortest Path, Communication of ACM 5, 345.

7. Garrison, W. Marble D., 1974. Graph theoretic concepts. Transportation Geography: Comments and Readings. McGraw Hill: New York, 58-80.

8. Gastner, M., Newman, M., 2006. The spatial structure of networks. The European Physical Journal B 49, 247-252.

9. Grubesic, T., Matisziw, T., Murray, A., Snediker, D. 2008. Comparative approaches for assessing network vulnerability. International Regional Science Review 31 (1), 88-112.

10. Hagget, P., Chorley, R., 1967. Models, paradigms and the new geography. Models in Geography, 19-41.

11. Holling, C., 1973. Resilience and stability of ecological systems. Annual Review of Ecology and Systematics 4, 1-23.

12. Jenelius, E., Mattsson, L., 2012. Road network vulnerability analysis of area-covering disruptions: a grid-based approach with case study. Transportation Research Part A 46, 746760.

13. Kansky, K., 1963. Structure of transport networks: Relationships between network geometry and regional characteristics, University of Chicago, Department of Geography, Research Paper \#84.

14. Laporte, G., Louveaux, F., 1993. The integer L-shaped method for stochastic integer problems with complete recourse. Operations Research Letters 13(3), 133-142.

15. Latora, V., Marchiori, M., 2002. Is the Boston subway a small-world network? Physica A Statistical Mechanics and its Applications 314, 109-113.

16. Miller-Hooks, E., Zhang, X., Faturechi, R., 2012. Measuring and maximizing resilience of freight transportation networks. Computers and Operations Research 39(7),1633-1643.

17. O'Kelly, M., 2014. Network hub structure and resilience. Resilience and vulnerability in spatial and economic networks. Networks and Spatial Economics (forthcoming).

18. Perna, A., Jost, C., Valverde, S., Gautrais, J., Theraulaz, G., Kuntz, P., 2008. The topological fortress of termites. Lect Notes Comput Sci 5151,165-173.

19. Reggiani, A., Bucci, P., Russo, G., 2011. Accessibility and impedance forms: empirical application to the German commuting network. International Regional Science Review 34 (2): $230-252$.

20. Reggiani, A., Nijkamp, P., Lanzi, D., 2015. Transport resilience and vulnerability: The role of connectivity. Transportation Research Part A (forthcoming).

21. Rodríguez-Núñez, E., García-Palomares, J., 2014. Measuring the vulnerability of public transport networks. Journal of Transport Geography 35, 50-63.

22. Rose, A., 2004. Defining and measuring economic resilience to earthquakes. Disaster Prevention and Management 13(4), 307-314.

23. Watts, D., Strogatz, S., 1998. Collective dynamics of `small-world' networks. Nature 393, 
440-442.

24. Weichselgartner, J., Kelman, I., 2014. Geographies of resilience: challenges and opportunities of a descriptive concept. Progress in Human Geography DOI: $10.1177 / 0309132513518834$.

25. Wu, J., Gao, Z., Sun, H., Huang, H., 2004. Urban transit as a scale-free network. Modern Physics Letters B 18, 1043-1049.

26. Yen, J., (1971). Finding the k shortest loopless paths in a network. Management Science 17 (11), 712-716.

27. Zhao, X., and Gao, Z., 2007. Topological effects on the performance of transportation networks. Chinese Physics Letters 24, 283. 